\title{
Gattini 2010: Cutting Edge Science at the Bottom of the World
}

Anna M. Moore*a, Sara Ahmed ${ }^{\mathrm{b}}$, Michael C. B. Ashley ${ }^{\mathrm{c}}$, Max K. Barreto ${ }^{\mathrm{d}}$, Xiangqun Cui ${ }^{\mathrm{e}}$, Alex

Delacroix $^{\mathrm{a}}$, LongLong Feng ${ }^{\mathrm{f}, \mathrm{g}}$, Xuefei Gong, ${ }^{\mathrm{e}, \mathrm{f}}$, Jon Lawrence, ${ }^{\mathrm{c}, \mathrm{h}}$, Daniel M. Luong-van ${ }^{\mathrm{c}}$, D.

Christopher Martin ${ }^{b}$, Reed Riddle ${ }^{a}$, Nicole Rowley ${ }^{i}$, Zhaohui Shang ${ }^{f, j}$, John W. V. Storey ${ }^{c}$, Nick F. H. Tothill ${ }^{c}$, Tony Travouillon ${ }^{b}$, Lifan Wang ${ }^{f, g, k}$, Huigen Yang ${ }^{f, 1}$, Ji Yang ${ }^{\mathrm{g}}$, Xu Zhou ${ }^{\mathrm{f}, \mathrm{m}}$, Zhengxi Zhu ${ }^{\mathrm{f}, \mathrm{g}}$

${ }^{a}$ Caltech Optical Observatories, 1200 E California Blvd, Pasadena, CA, USA 91125;

${ }^{\mathrm{b}}$ California Institute of Technology, 1200E California Blvd, Pasadena, CA, USA 91125 ;

'School of Physics, University of New South Wales, Sydney NSW 2052, Australia;

${ }^{d}$ Hawai'i Preparatory Academy, 65-1692 Kohala Mtn. Rd., Kamuela, HI 96743, USA;

${ }^{\mathrm{e} N a n j i n g ~ I n s t i t u t e ~ o f ~ A s t r o n o m i c a l ~ O p t i c s ~ \& ~ T e c h n o l o g y, ~ N a n j i n g ~ 210042, ~ C h i n a ; ~}$

${ }^{\mathrm{f}}$ Chinese Center for Antarctic Astronomy, China;

g Purple Mountain Observatory, Nanjing 210008, China;

${ }^{\mathrm{h}}$ Australian Astronomical Observatory, Sydney NSW 1710, Australia;

${ }^{i}$ University of California, Los Angeles; 405 Hilgard Ave, Los Angeles CA 90095 USA, USA;

${ }^{\mathrm{j}}$ Tianjin Normal University, Tianjin 300074, China;

${ }^{\mathbf{k}}$ Department of Physics and Astronomy, Texas A\&M University, College Station 77843, USA;

${ }^{1}$ Polar Research Institute of China, Shanghai 200136, China;

${ }^{\mathrm{m}}$ National Astronomical Observatories, Chinese Academy of Science, Beijing 100012, China.

\begin{abstract}
The high altitude Antarctic sites of Dome A and the South Pole offer intriguing locations for future large scale optical astronomical Observatories. The Gattini project was created to measure the optical sky brightness, large area cloud cover and aurora of the winter-time sky above such high altitude Antarctic sites. The Gattini- DomeA camera was installed on the PLATO instrument module as part of the Chinese-led traverse to the highest point on the Antarctic plateau in January 2008. This single automated wide field camera contains a suite of Bessel photometric filters (B, V, R) and a long-pass red filter for the detection and monitoring of $\mathrm{OH}$ emission. We have in hand one complete winter-time dataset (2009) from the camera that was recently returned in April 2010.

The Gattini-South Pole UV camera is a wide-field optical camera that in 2011 will measure for the first time the UV properties of the winter-time sky above the South Pole dark sector. This unique dataset will consist of frequent images taken in both broadband $\mathrm{U}$ and $\mathrm{B}$ filters in addition to high resolution ( $\mathrm{R} \sim 5000)$ long slit spectroscopy over a narrow bandwidth of the central field. The camera is a proof of concept for the $2 \mathrm{~m}$-class Antarctic Cosmic Web Imager telescope, a dedicated experiment to directly detect and map the redshifted lyman alpha fluorescence or Cosmic Web emission we believe possible due to the unique geographical qualities of the site.
\end{abstract}

We present the current status of both projects.

Keywords: Gattini, Dome A, PLATO observatory, Antarctic wide field surveys, cosmic web, site testing, cloud cover, aurora, night sky brightness

\section{GATTINI-DOME A}

The Gattini-Dome A camera ${ }^{1}$ was deployed to Dome A in the Antarctic summer season of 2008/2009 as part of an international effort led by the Polar Research Institute of China as part of the Chinese PANDA International Polar Year project ${ }^{2}$. 


\subsection{Project goals}

The goals of the Gattini-DomeA project are as follows:

1. To obtain the night sky brightness in $\mathrm{B}, \mathrm{V}$, and $\mathrm{R}$ photometric bands above Dome A site;

2. To measure the cloud cover extent during the 2009 winter season and onwards;

3. To provide aurora and airglow statistics combined with spectra obtained with the NIGEL instrument ${ }^{3}$;

4. Perform Photometry of bright target stars in field with an unprecedented window function permitted by the continuous winter conditions.

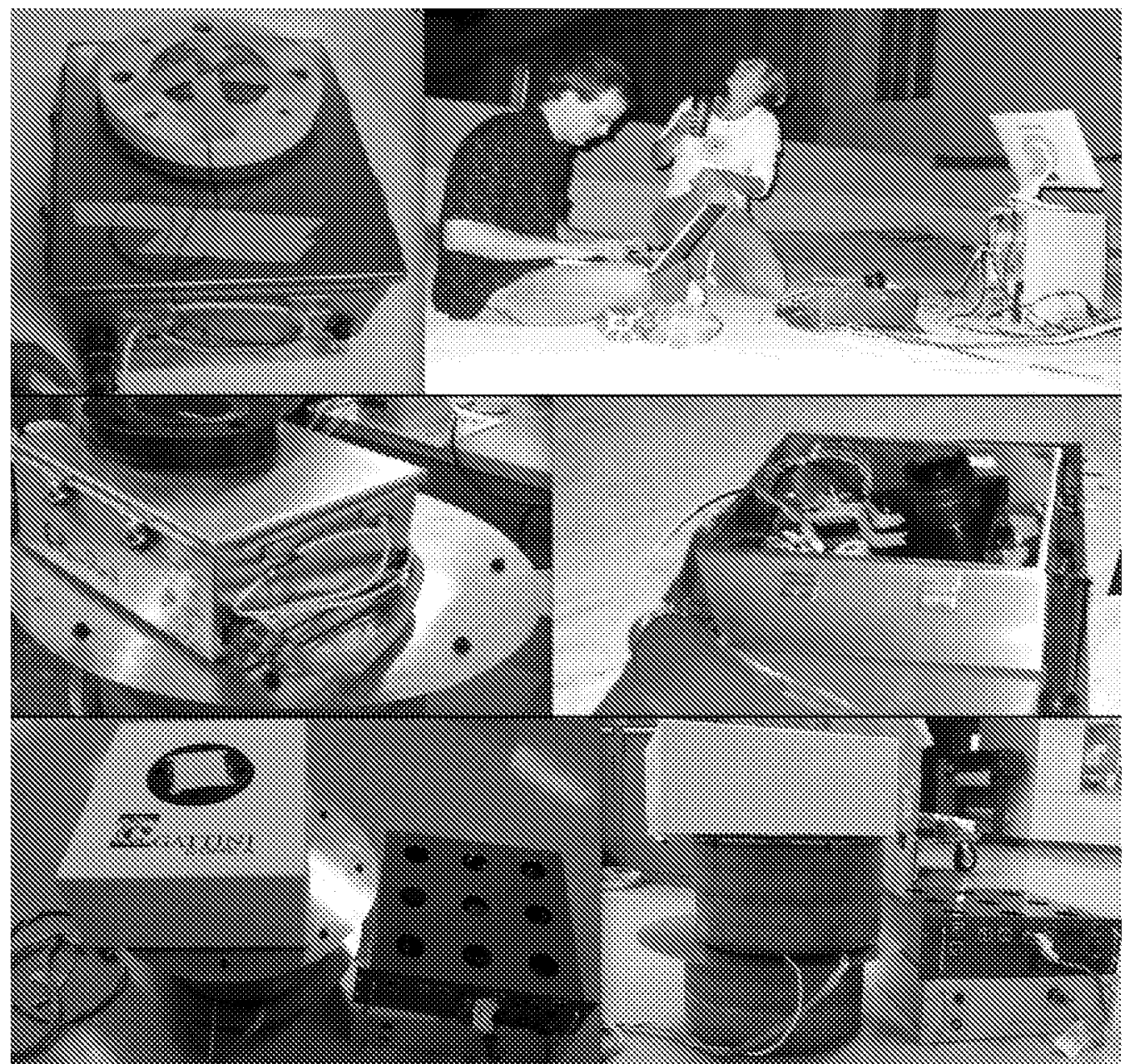

Figure 1: The Gattini-Dome A experiment under assembly and testing at Caltech in 2008

\subsection{Dome A Camera design and assembly}

The camera consists of a Nikon 10.5mm f/2.8G ED DX Fisheye Lens mated to an Apogee Alta U4000 2k x 2k interline camera. The system results in a sampling of $\sim 2.4 \operatorname{arcmin} /$ pixel or $90^{\circ} \times 90^{\circ}$ field of view. Located in between the lens and camera is a 5-position filter wheel containing Bessel B, V, and R filters. The remaining two slots are for a long pass red filter $(>650 \mathrm{~nm})$ for the study of airglow emission and an opaque mask for dark current tests.

The camera assembly is located inside a small heated enclosure as shown in Figure 1 and 2 (left). The heat permits the successful operation of the off-the-shelf camera as well as eradicating ice formation on the window surface. Conductive Indium Tin Oxide coating was employed on the window surface as a method of de-icing however it was found that when operational this was not sufficient to remove the newly formed ice. 

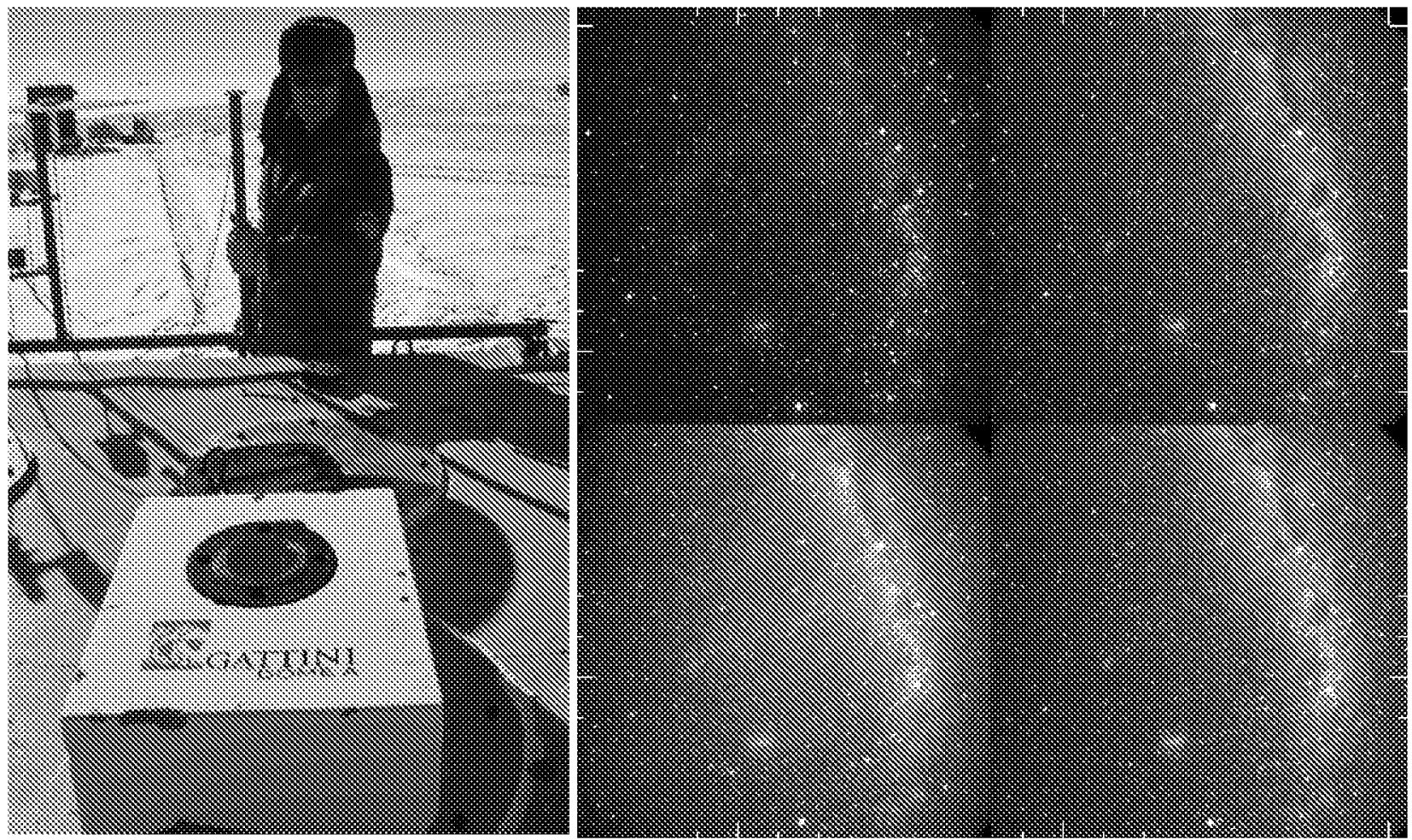

Figure 2: (left) The Gattini camera at Dome A located on the roof of the PLATO Observatory; (right) 4x100s exposures taken in dark time for each of the four filter bands $[\mathrm{TL}=\mathrm{B}, \mathrm{TR}=\mathrm{R}, \mathrm{BR}=\mathrm{R}, \mathrm{BL}=\mathrm{OH}]$.

The experiment is controlled by a low power PC located in an electronics rack inside the PLATO ${ }^{2}$ module. The continuous observation sequence is operationally simple and is as follows:
1. $100 \mathrm{~s} \mathrm{~B} 30 \mathrm{~s} \mathrm{~B}$
2. $100 \mathrm{~s}$ V $30 \mathrm{~s} \mathrm{~V}$
3. 100 s R 30 s R
4. $100 \mathrm{~s} \mathrm{OH} 30 \mathrm{~s} \mathrm{OH}$
5. 100s dark, BIAS frame
6. REPEAT

During periods of bright twilight at the beginning and end of the season, the exposure time is adjusted to adapt to the median ADU count to prevent over-exposure.

\subsection{Dataset}

A limited but useful set of data was downloaded during the 2009 winter season via the Iridium satellite network. The full dataset returned to Caltech in mid-April 2010 . The dataset contains $\sim 160,000$ images equating to $\sim 1000 / 24$ hours. The start date is April $18^{\text {th }} 2009$ and finish date is October $10^{\text {th }} 2009$. The time interval between each exposure is roughly $1-2$ minutes.

\subsection{Sky Brightness}

The uncalibrated sky brightness in each of the filter bands is shown in Figure 3. The counts are detector ADU/sec/pixel. The monthly variation due to the moon is noted in addition to the daily diurnal variation due to solar contribution. The dashed horizontal line indicates the approximate detector flux required for a B band sky brightness of $21.6 \mathrm{~B}$ magnitudes/arcsec ${ }^{2}$. Further in depth data reduction of the full dataset is required to confirm this minimum value. 


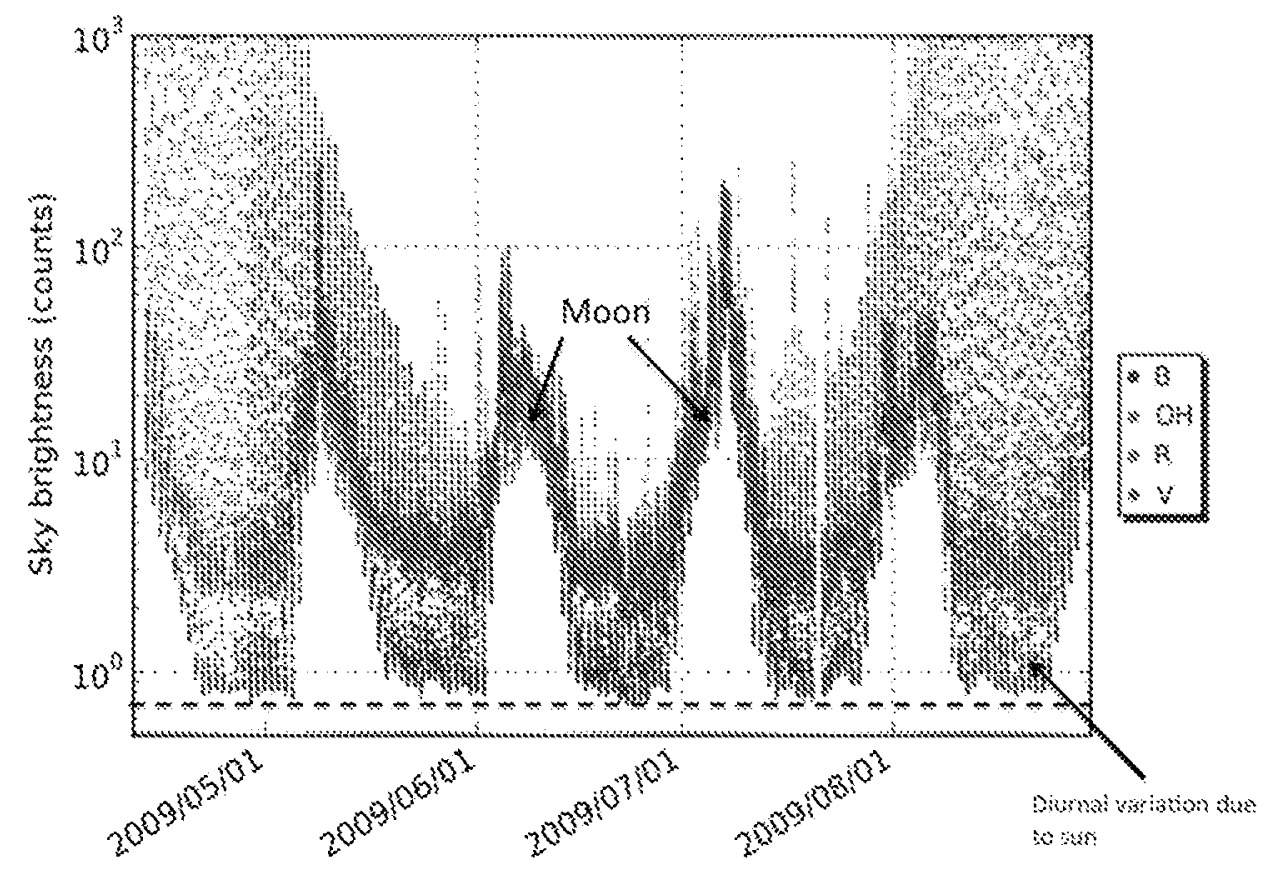

Figure 3: Uncalibrated sky brightness values in B, V, R and long pass red 'OH' filters throughout the 2009 winter season. The dashed horizontal line gives the approximate sky brightness reading that equates to a B band magnitude of 21.6 $\mathrm{mag} / \operatorname{arcsec}^{2}$.

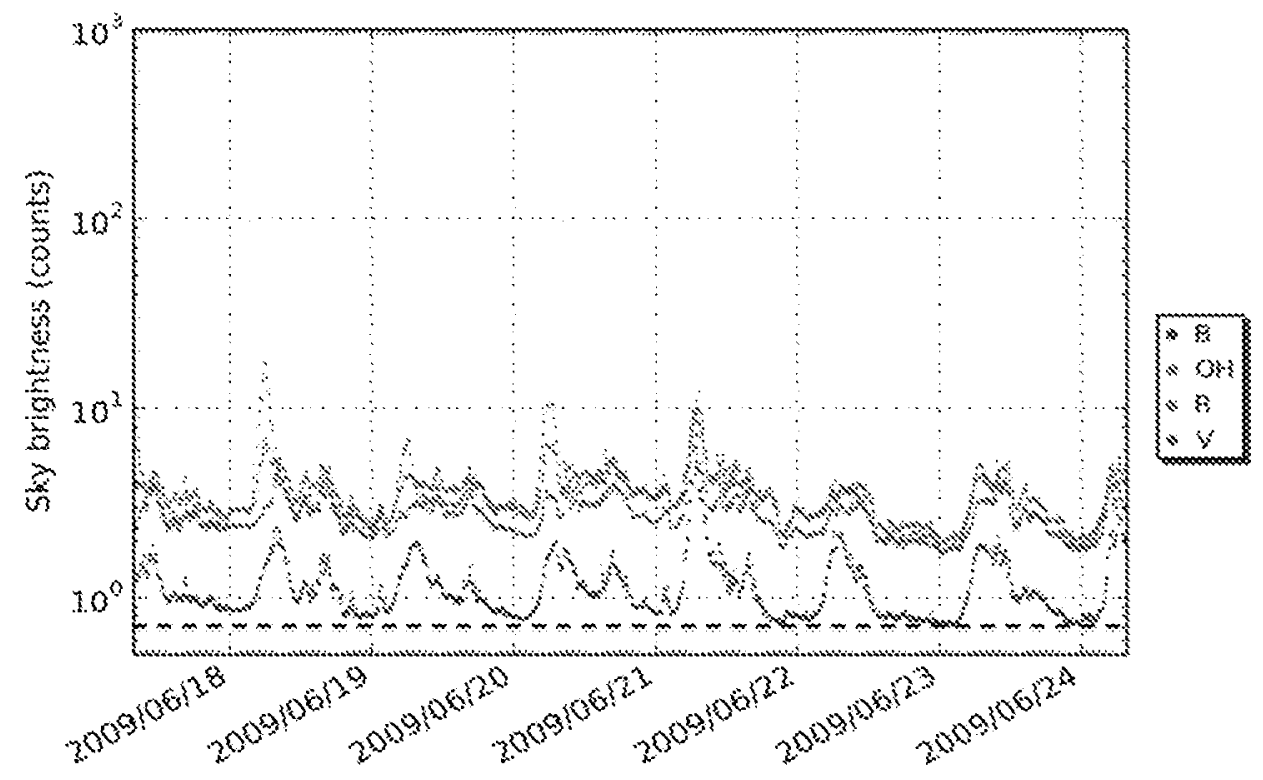

Figure 4: Above plot zoomed to one week duration centered on mid-winter 2009 


\subsection{Photometry}

Preliminary results of the possible photometric accuracy are shown in the figures of Figure 5. The left figure shows the raw relative magnitude difference between 2 stars in the field over a period of 2 months. The stars were deliberately chosen for their relatively constant magnitude and closeness on the sky. Only overscan and sky background were subtracted. We see a performance of $\sim 10-20 \mathrm{mmag}$ rms for the stars under analysis. The right-hand figure of Figure 5 shows the same results for L Car (HR3884), a Galactic Cepheid star of apparent V magnitude $\sim 4-5$ that is the brightest Cepheid in the sky.
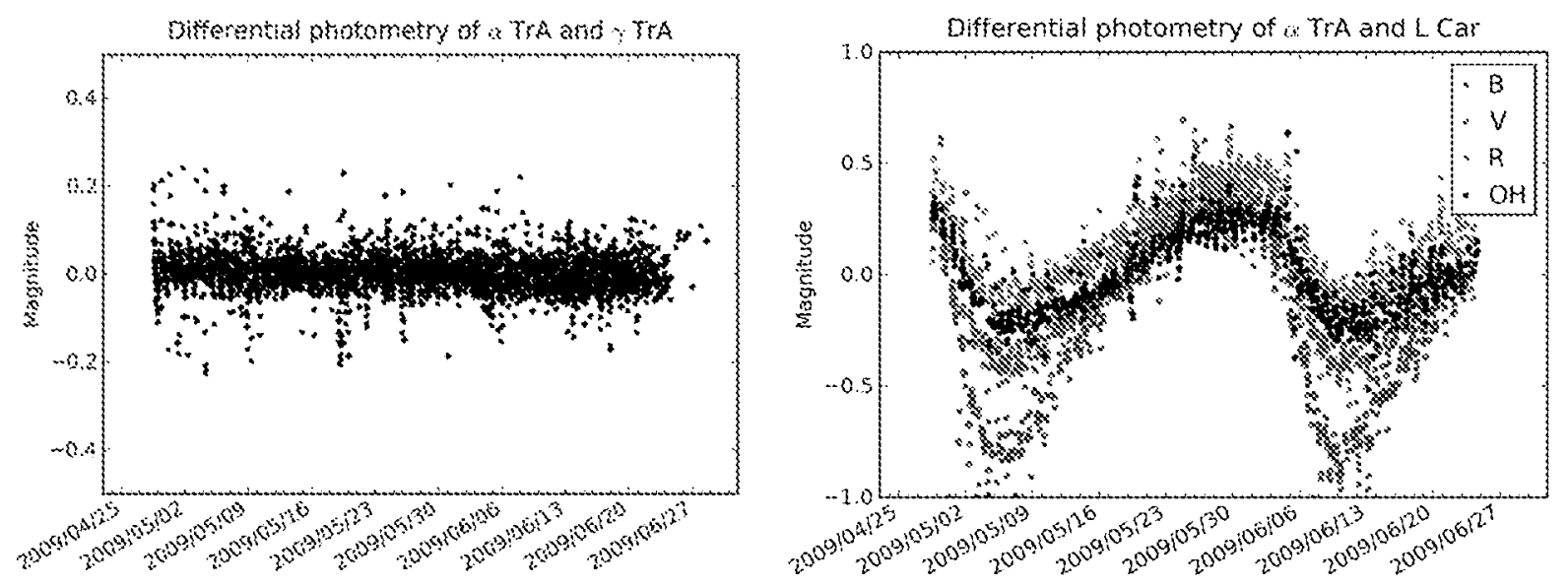

Figure 5: (left) Differential photometry of two close stars in the Gattini field show an accuracy of $\sim 10-20 \mathrm{mmag}$ rms, without flat fielding or further tools for analysis; (right) the B,V,R and OH light curves for the brightest Cepheid star in the sky L Car (HR3884).

\subsection{Status}

The full dataset is currently under analysis. The deliverables of the analysis are as follows (1) calibrated sky brightness values in mag/arcsec ${ }^{2}$ for each of the Bessel filter bands for the 2009 winter season; (2) statistics for the measured cloud cover in particular the median continuous observation period; (3) in combination with the NIGEL medium resolution experiment derive statistics for the aurora contribution in particular frequency, wavelength and equivalent sky background contribution; (4) produce light curves for the brightest targets in the field and; (5) detect possible transient events such that software can be developed for future seasons when quick notification will be of interest.

\section{GATTINI-SOUTH POLE UV EXPERIMENT}

\subsection{Introduction}

The Gattini South Pole UV experiment will characterize the South Pole winter sky from 2011 onwards in the Astronomical $\mathrm{U}$ and $\mathrm{B}$ bands to provide a foundation for future larger-scale experiments such as the Antarctic Cosmic Web Imager 5 . The science case of ACWI requires the direct detection of Lya emission of the Intergalactic Medium (IGM). As such the experiment requires exquisite subtraction of the night sky background in order to detect the extended and faint emission from the targets. A South Pole location is identified for this purpose given (1) the target elevation is constant; (2) instrumental contributions to inaccurate sky subtraction such as flexure and temperature variation can be eliminated and; (3) the possibility of observing at wavelengths approaching $300 \mathrm{~nm}$ from this Antarctic location. However, time varying emission from the sky such as aurora could be potentially damaging to this specific science case due to a South Pole location, therefore, the Gattini SPUV experiment was proposed and funded to perform a pathfinder analysis of the site.

\subsection{Goals}

The experiment goals are to: 
1. Characterize the South Pole winter sky in the Astronomical U and B bands to provide a foundation for future larger-scale experiments such as the Antarctic Cosmic Web Imager (direct detection of Lya emission of the Intergalactic Medium (IGM));

2. Provide fundamental data for other leading-edge science cases that require virtually no instrument-induced noise, exquisite background subtraction, and are invariant to image quality at these wavelengths;

3. Measure the atmospheric extinction in the $U$ and $B$ bands from a zenith angle of 0 to 45 degrees;

4. Obtain frequent and wide field observations of two of the brightest airglow lines occurring in the $U$ and $B$ bands, and;

5. Produce frequent and wide field observations of two of the brightest aurora lines in the $U$ and $B$ bands.

\subsection{Design methodology}

Three designs of differing complexity and scientific return are summarized in Table 1. The proposed GOAL design of the Gattini South Pole UV camera includes the following:

1. The Gattini-SPUV Imager: A fixed orientation (transit) imager with an aperture of at least $100 \mathrm{~mm}$ and field of view $4^{\circ} \times 4^{\circ}$, that includes at least 2 filters ( $U$ and $B$ at a minimum), aligned to the South Pole;

2. The Gattini-SPUV Spectrograph: A fixed orientation spectrograph channel of at least $100 \mathrm{~mm}$ aperture and field of view 2 deg with spectroscopic resolution of at least $R \sim 5000$, centered at $\sim 350 \mathrm{~nm}$ (TBD), with a single slit of appropriate width located at the intermediate focal plane, system is aligned to the South Pole and;

3. Wide field images taken by the South Pole All-sky imager ${ }^{4}$ using the $589 \mathrm{~nm}$ filter and long exposure time in a collaboration between Caltech and the All-sky imager team. The resulting dataset will be used to determine cloud cover statistics at the site.

Table 1

\begin{tabular}{|c|c|c|c|}
\hline \multirow{2}{*}{\multicolumn{4}{|c|}{ TECHNICAL CAPABILITY }} \\
\hline & & & \\
\hline 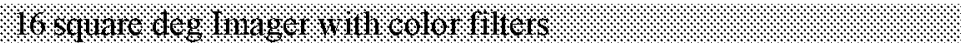 & 3 & & \\
\hline $\begin{array}{l}16 \text { square deg Imager with interference filters, requires large filter } \\
\text { wheel or extra optics for collimated beam }\end{array}$ & $\mathrm{X}$ & $\mathrm{X}$ & \\
\hline  & X. & & \\
\hline 90 deg B band imager & $\mathrm{X}$ & & \\
\hline  & a. & :ı: & ৭. \\
\hline 16 square deg Imaging dataset $\mathrm{U}$ band & $\mathrm{X}$ & $\mathrm{X}$ & $\mathrm{X}$ \\
\hline 16 square deg lmaging dataser B band. & 1. & \%. & \%. \\
\hline $\mathrm{R}=5000$ spectroscopy of 2 deg slit length, fixed bandwidth & $\mathrm{X}$ & $\mathrm{X}$ & \\
\hline m. & & $x$ & \\
\hline $\begin{array}{l}\text { Wide field images B band, } 90 \text { deg FOV, cloud cover and B band } \\
\text { extinction only }\end{array}$ & $\mathrm{X}$ & & \\
\hline
\end{tabular}

\subsection{Experiment design}

The ccd detector format is the starting point for the optical design. The requirements are to image a $4^{\circ}$ field of view onto the detector ccd of choice, with a rms image size of one pixel across the field, from $300 \mathrm{~nm}$ to $500 \mathrm{~nm}$ without the need to refocus.

\section{Detector}

The detector of choice is the iKon-M 934 from Andor Technology. Two identical cameras have arrived at Caltech and are under detector charaterization testing. The CCD is a $1 \mathrm{k} \mathrm{x} 1 \mathrm{k}$ back-illuminated device with $13 \mathrm{um}$ pixels. The 2-stage peltier cooler obtains a minimum detector temperature of $-80^{\circ} \mathrm{C}$ without water-cooling. 


\section{GOAL Design Optical layout}

The optical layout of the most ambitious but most scientifically productive Gattini-UV design is shown in Figures 6 . The system aperture is 6 in diameter and the assembly fits inside a volume of $\sim 35 \times 35 \times 24$ inches. The optical design consists of: (1) a 6in aperture Telescope based on a Schmidt design with aspheric corrector plate that images $4^{\circ} \times 4^{\circ}$ field at Cassegrain focus; (2) the focus contains 2 fold mirrors - one to divert light to the imager and one to send a slit image to the spectrograph; (3) for the imager channel a fast $\mathrm{f} / 1.53 \mathrm{Schmidt}$ camera images the $4^{\circ} \mathrm{x} 4^{\circ}$ image to the Andor CCD; (4) the imager channel contains a simple spherical collimator for imaging of the pupil at the 4-position filter wheel; (5) the spectrograph channel incorporates a slightly slower Schmidt camera for reimaging a $2^{\circ}$ long slit to the Andor CCD that is spectrally dispersed at a resolution of $\mathrm{R} \sim 5000$; (6) a static Volume Phase Holographic grating replaces the filter wheel in the spectrograph channel.

The secondary obscuration is $\sim 25 \%$. There are 15 optical elements of which 3 are aspheric, 3 are conic and the remaining items are either flat or spherical. A tolerance analysis was performed that has been incorporated into the mechanical design of the experiment.

The optical layout is shown in Figure 6 with rendered mechanical drawings shown in Figures 8 and 9.

\section{Performance}

Optical performance of the imager is shown in Figure 7. The imaging performance is excellent over the field with 2pixel sampling of the PSF across the $4^{\circ}$ field of view. Distortion is $0.06 \%$. The imager will be able to detect point sources to a minimum magnitude of 16 Bmag as shown in Figure 6.

\section{Materials consideration}

All optics, refractive or otherwise, are made of UV grade fused silica. Fused silica has excellent transmission at UV wavelengths and, in addition, has a low thermal coefficient of expansion. For these reason the custom reflective optics will be made of low expansion glass (zerodur or fused silica for example) where possible.

\section{Shutter}

Acquisition of a frame transfer ccd was not possible from Andor Technology. Therefore we require a system shutter, likely located just ahead of the detector window. A low risk solenoid type has been identified as a possible solution

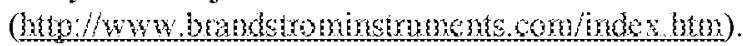

\begin{tabular}{|c|c|c|c|c|c|c|c|c|c|c|}
\hline \multicolumn{2}{|c|}{ 3.13: } &  & & & & & & & & \\
\hline \multicolumn{2}{|c|}{ 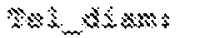 } & $32 \leqslant n$ & & & & & & & & \\
\hline \multicolumn{2}{|c|}{ 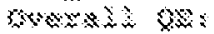 } & 3.2 & & & & & & & & \\
\hline \multicolumn{2}{|c|}{ 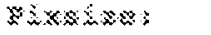 } & 30 & $(2-3003)$ & & & & & & & \\
\hline \multicolumn{2}{|c|}{ Resdintine } & 2.510 & conses & & & & & & & \\
\hline \multicolumn{2}{|c|}{ 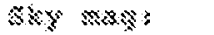 } & $28 \leqslant 2$ & $2 x^{2} 2 x \in 0$ & & & & & & & \\
\hline \multicolumn{2}{|c|}{$\operatorname{sing} \theta$} & 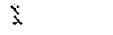 & & & & & & & & \\
\hline \multicolumn{2}{|c|}{$\$<\&, \alpha 2<E$} & 3. & & & & & & & & \\
\hline \multicolumn{2}{|c|}{ Moptikn: } & $6 \%$ & & & & & & & & \\
\hline \multicolumn{2}{|c|}{ Ww: } & $\sum \quad 3 \times \infty$ & & & & & & & & \\
\hline \multicolumn{2}{|c|}{ 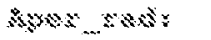 } & 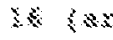 & & & & & & & & \\
\hline 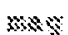 & (6.30; & 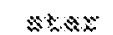 &  & sisy & $\Sigma s$ & $\dddot{x} \geqslant \infty$ & w & $\ldots$ & $\approx / \mathrm{N}$ & 3208.23 \\
\hline$m e x$ & ?. 803 & 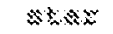 & 30384 & $\$ y$ & 268 & 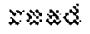 & 3 & $\ldots$ & 㐘 & 365 \\
\hline$x$ & 0,80 & $s+8 x$ & $2316 \leqslant$ & $x y$ & $28 \theta$ & $x+2$ & 3 & $\omega$ & Si & 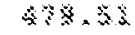 \\
\hline $\max$ & $\$, 80$ & 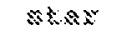 & $8 \times 3$ & $\approx 8 y$ & $26 \%$ & $x^{2}=2 \mathrm{a}$ & 28 & 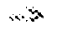 & sid & 260,28 \\
\hline $3 \times 8$ & 30,020 & 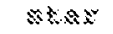 & 3872 & 3. & $28 \%$ & 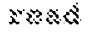 & 20 & $\cdots$ & Sid & 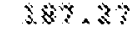 \\
\hline $3 \times$ & 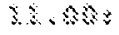 & $\Leftrightarrow \& A x$ & 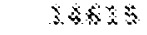 & $\$ 8$ & $2 x$ & 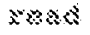 & 20 & $\ldots$ & $s i x$ & 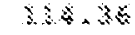 \\
\hline$m a x$ & $32,8 \%$ & 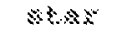 & $5 \alpha$ & $\$ \mathrm{Ny}$ & 368 &  & 28 & $\ldots$ & 8 & 8,2 \\
\hline $\operatorname{sis}$ & $\$ 3.00 x$ & $\otimes B \times x$ & 2306 & $s \mathrm{~s}$ & 368 & $2 \times 20$ & 20 & $\ldots$ & $68 \mathrm{x}$ & $38 . \leqslant 5$ \\
\hline $\operatorname{mos}$ & $5.08 \mathrm{x}$ & $\$ 3: 8$ & 823 &  & 308 & stases & 20 & $\cdots$ & $S \mathrm{~N}$ & 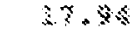 \\
\hline sise & 5,04 & $\operatorname{sen} 5$ & $35 ?$ & $\$ N y$ & 38 & Feas & 20 & $\cdots$ &  & $\$+\beta$ \\
\hline$m$ & S. 0 : & $\operatorname{san}=$ & 30 &  & 30 & $50 x$ & 20 & $\cdots$ & 50 & $3+3 \times$ \\
\hline
\end{tabular}

Figure 6: Performance estimate for the Gattini-SPUV $4^{0}$ imager. 


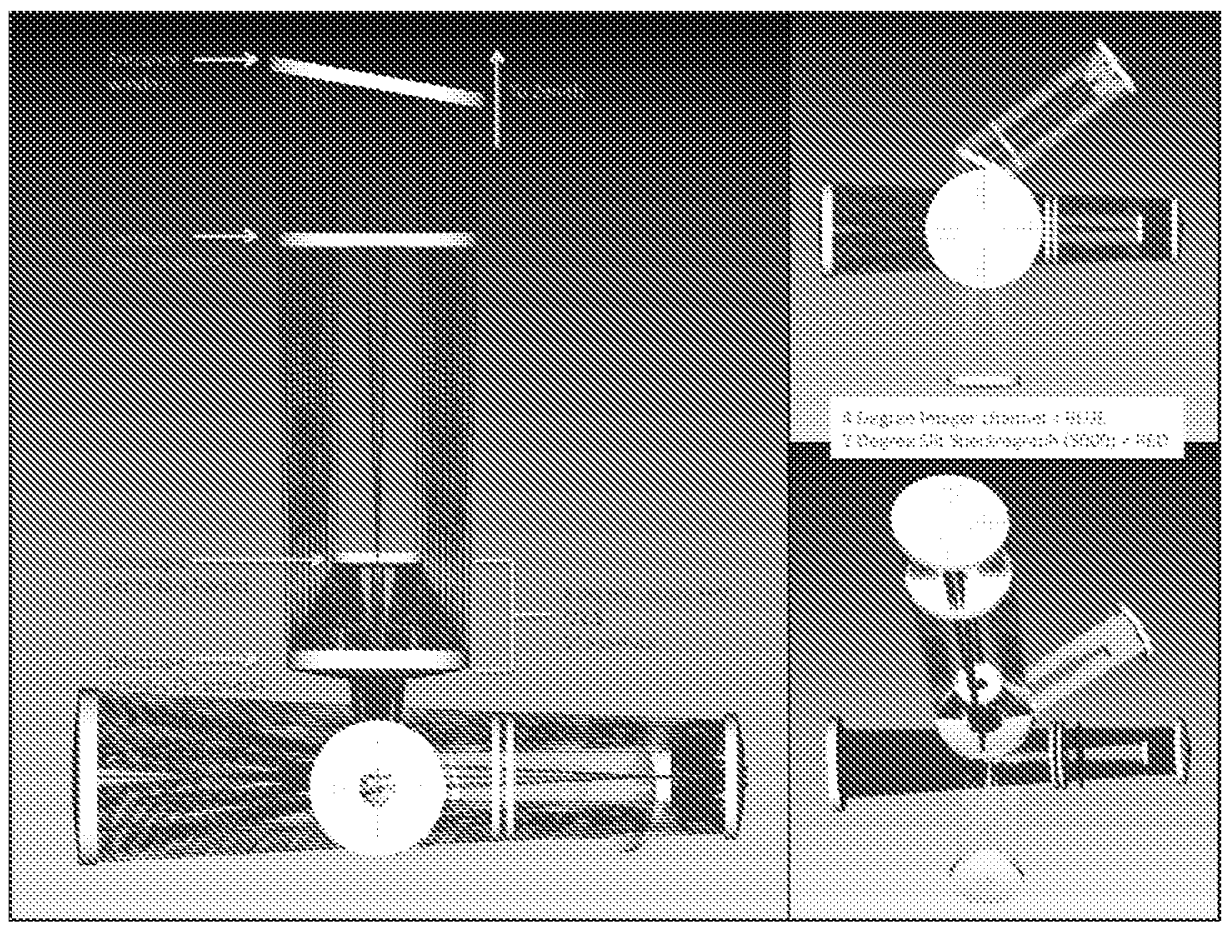

Figure 7: Optical layout of the double channel Gattini-SPUV experiment

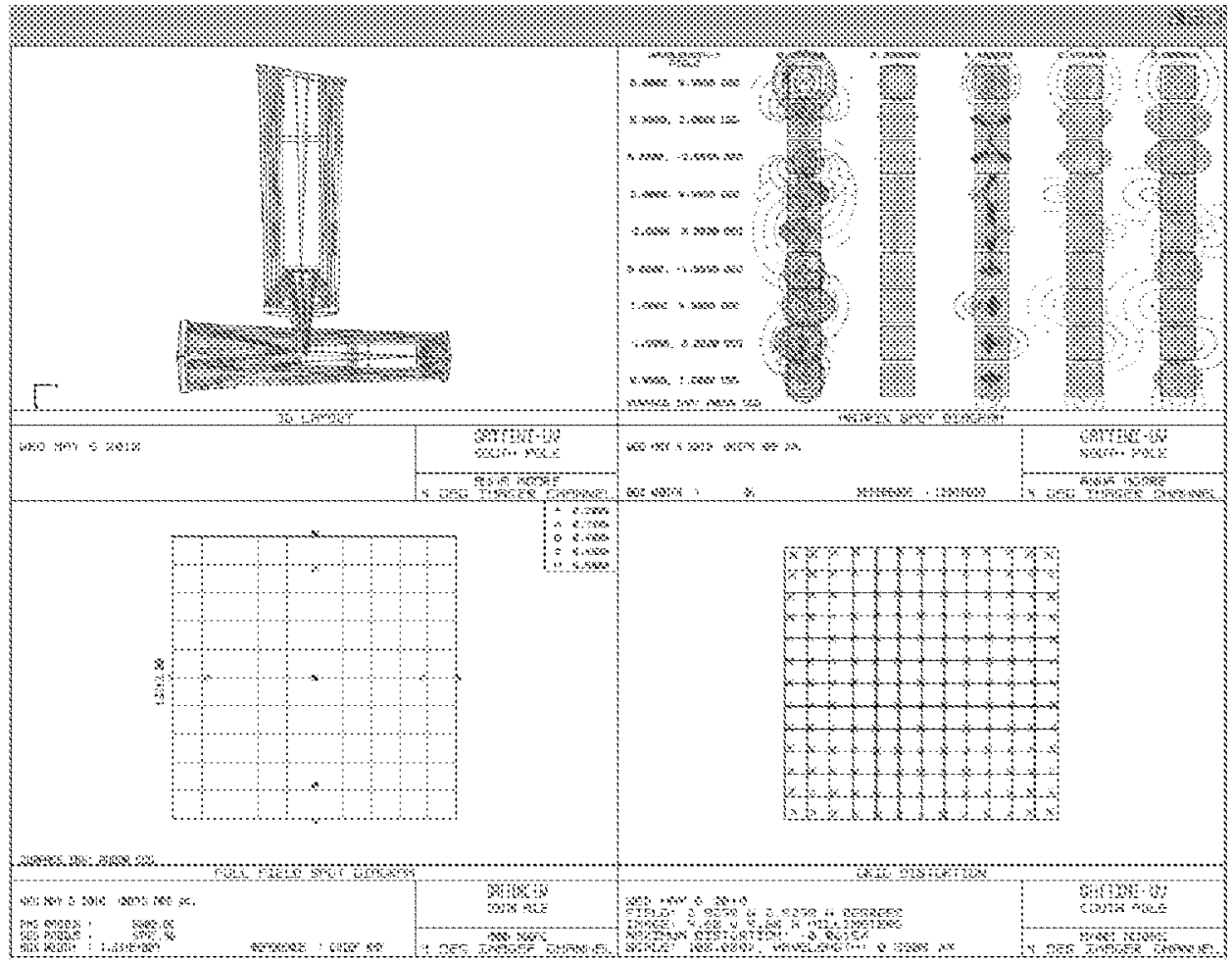

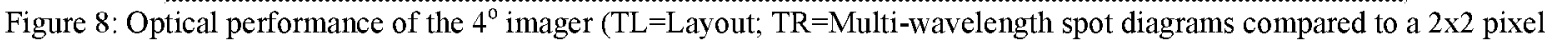
box; $\mathrm{BR}=$ Distortion plot; $\mathrm{BL}=$ Field positions used in analysis). 


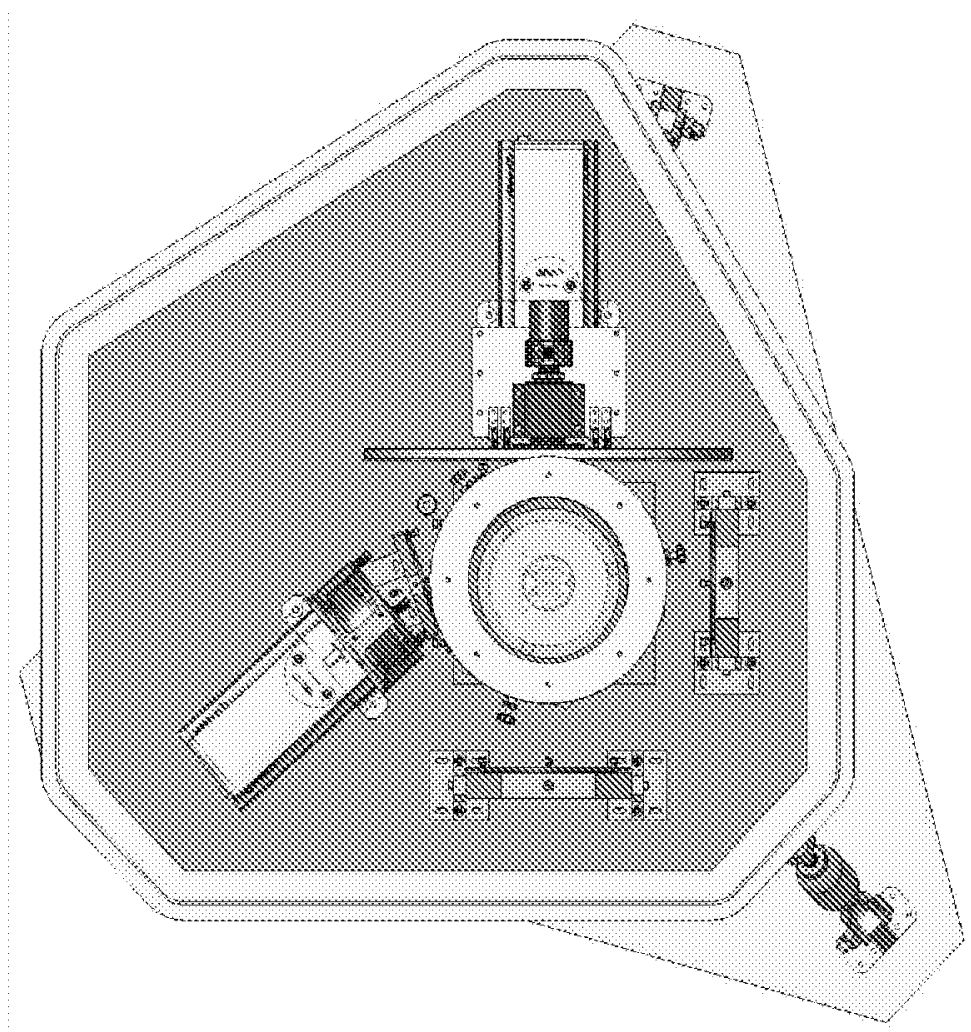

Figure 9: Plan view of the Gattini-SPUV experiment

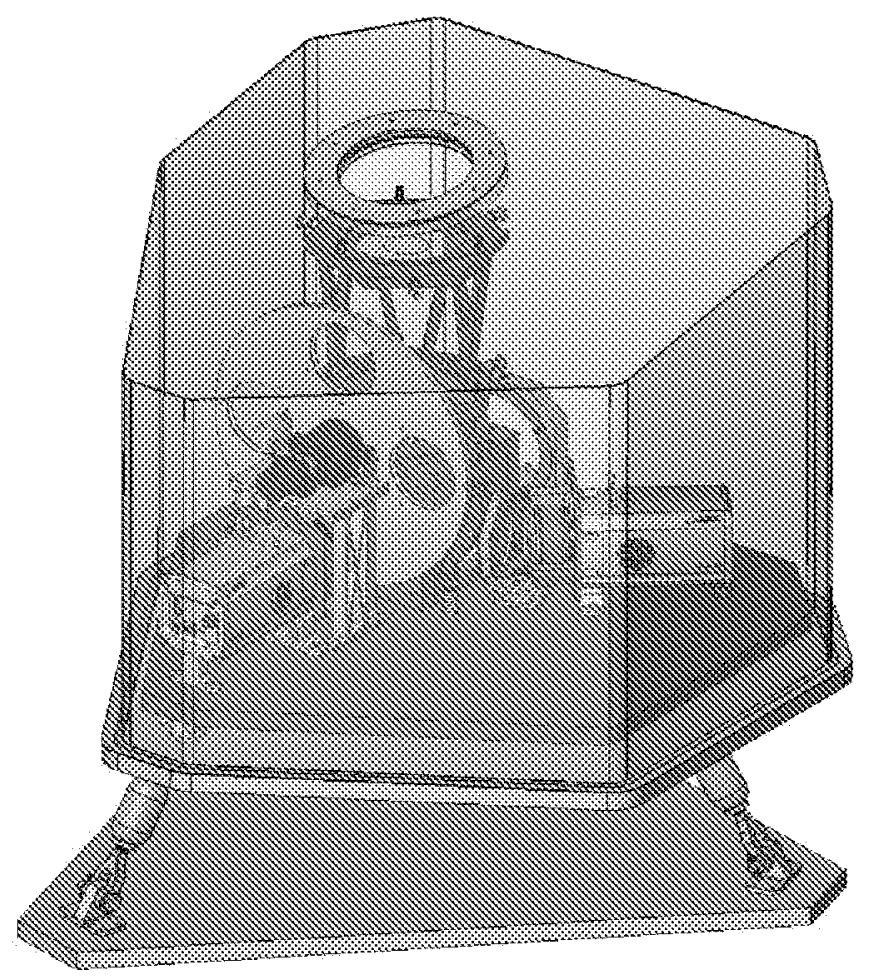

Figure 10: Isometric view of the Gattini-SPUV experiment inside the enclosure 


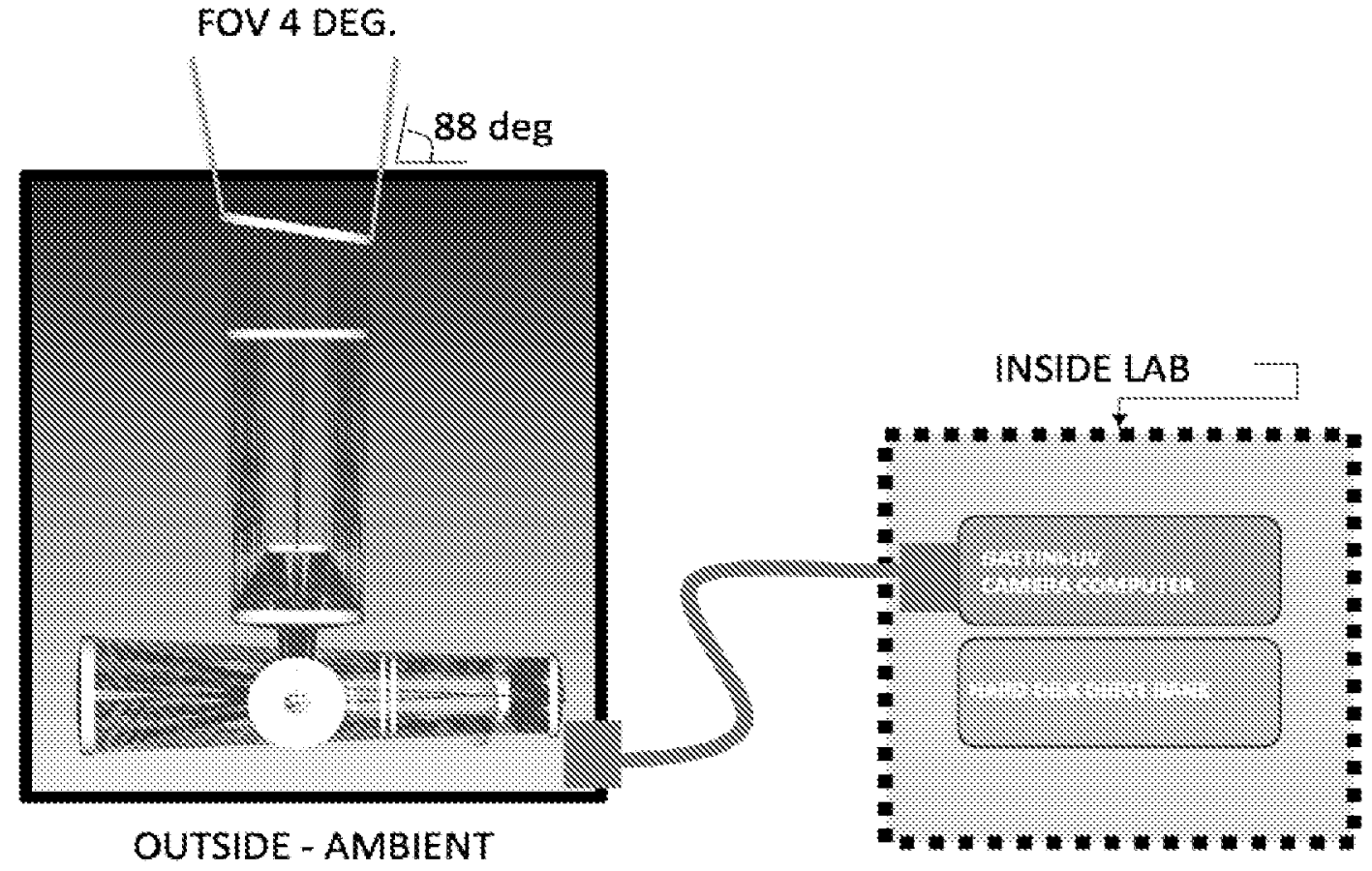

Figure 11: Schematic of the control pc and linkage to the experiment located at ambient

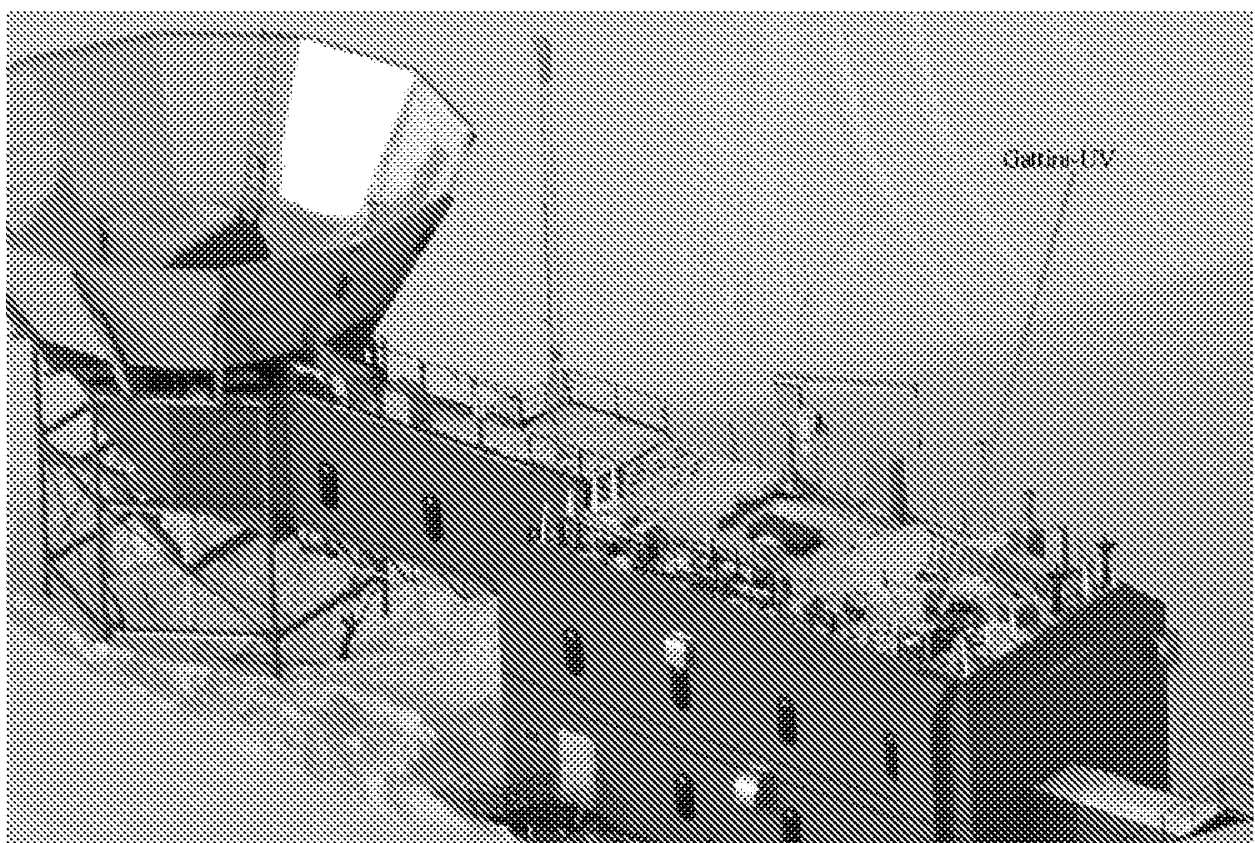

Figure 12: Location of the Gattini-SPUV experiment will be on the roof of the MAPO building located in the dark sector of the South Pole Amundsen Scott station 


\subsection{Fabrication, assembly and verification}

\section{Working temperature}

The operating temperature range for the Gattini-UV enclosure is $+25^{\circ} \mathrm{C}$ (lab) to $-75^{\circ} \mathrm{C}$ (minimum at South Pole). The control electronics will be housed in a heated laboratory while operating at the South Pole so does not need to sustain the above temperature minimum.

Enclosure The cameras should operate successfully at ambient temperature at the South Pole. Given this, we have chosen not to maintain the internal temperature of the enclosure, but rather let it float with the external ambient temperature.

The starting principle is to have a sealed and therefore airtight enclosure that is flushed with dry Nitrogen at the beginning of the winter season. The enclosure does not need to be vacuum rated but must be capable of withstanding small pressure differences created by the varying ambient pressure. This approach is identical to that adopted in the successful CSTAR [ref] campaign at Dome A. All optics and opto-mechanical assemblies require mounting to an invar bench so that no realignment or refocus is required from lab to low temperature operation of the experiment.

De-Icing/Ice prevention Icing is always an issue to consider when designing an optical telescope for Antarctica. Optics such as entrance windows stare at the sky and are, therefore, usually the coldest part of the instrument. Without heating of some sort the surface temperature can quite easily drop below the dew point.

(1) Internal optics The approach here is to remove all water from the internal enclosure and then prevent it from entering thereafter. If successful, this means we do not have to worry about icing on internal optics. In addition, we can add a strong desiccant such as Calcium Hydride and a cold finger that links the outside ambient temperature to the internal enclosure. This permits a surface inside the enclosure to drop to, possibly, the coldest surface temperature inside the enclosure. This would be especially good in cases of a strong drop in ambient temperature.

(2) External window A successful method of ice prevention adopted in the $\operatorname{CSTAR}^{6}$ campaign was to coat the entrance window with Indium Tin Oxide (ITO), a conductive coating that is transparent at visible wavelengths. The coating layer increases in temperature when a current is passed through it, so allowing the coated surface to be raised above the dew point by more than 3 degrees (necessary for ice prevention).

Regrettably, ITO does not transmit well at UV wavelengths and cannot be used without a substantial loss in throughput $(\sim 22 \%$ transmission at $300 \mathrm{~nm}, \sim 35 \%$ transmission at $350 \mathrm{~nm})$. Instead, we have chosen to heat the external window to prevent icing and/or remove ice if formed. The window is orientated at an angle of $\sim 15^{\circ}$ and care will be taken to not to have sharp surfaces close to the window that can offer convenient places for ice accumulation. It should be noted that the South Pole does not suffer from the extreme surface ice accumulation that occurs at the higher sites of Dome A and Dome C.

Electrical The Gattini-UV enclosure, located at external ambient temperature, is linked to a rack-mounted control computer by necessary low temperature rated cables. This is the design layout adopted in all previous Gattini camera systems. MILSPEC connectors will be used throughout the design. A vacuum rated connector will be incorporated into the ambient enclosure. Low temperature rated cabling (PTFE) will be used throughout the design.

Mechanisms The GOAL design has three incorporated mechanisms: (1) 2 shutters (likely located in front each Andor CCD housing) and; (2) a low temperature filter wheel, housing up to 4 filters of diameter 4 " (with minimum of 2). The filter wheel is considered high risk and as such we have approached a local company that specialize in the manufacture and assembly of low temperature mechanisms.

Testing Low temperature testing of the entire instrument is considered a mandatory requirement. To aid this the project has purchased a low temperature environmental chamber to be located in the clean room of the Cahill Astronomy and Astrophysics lab. The experiment, time permitting, will be tested on sky at Palomar Observatory, roughly 2.5 hours drive from Caltech. This will aid greatly in the data reduction software development and provide an excellent comparison data set from an operational Observatory site with usually low background in the "blue".

South Pole Alignment We require the imager field of view to be aligned closely to the South Pole rotation axis. In the past this alignment has almost always been performed during the summer months using the sun, given the remoteness of the sites in question. This can be performed again to adequate accuracy at the South Pole, with the possibility of readjusting the alignment during the winter if required. The enclosure requires a method of tip and tilt adjustment. The 
most likely solution is to have an adjustable tripod support. The experiment will be located on the roof of the MAPO building in the dark sector at the South Pole station as shown in Figure 12.

Gattini-SPUV Status The timeline for the experiment is as follows: (1) June 2010 Sara Ahmed (undergraduate student) arrives to commence camera testing; (2) June 2010 Nicole Rowley (undergraduate student) arrives to commence data reduction of previous experiment to tune design of Gattini SPUV data reduction software; (3) June 2010 Finalize optics orders; (4) July 2010 Finalize mechanical drawings and send to manufacturer; (5) August 2010 Environmental chamber arrives; (6) August/September 2010 Receive control computer from UNSW; (7) August-October 2010 Assemble instrument and cold test; (8) November 2011 (time permitting) Site test at Palomar Observatory; (9) Dec 2010 Instrument packed and shipped to South Pole; (10) Jan/Feb 2011 Two team members arrive for installation of experiment at Amundsen Scott station at South Pole; (11) April 2011 Commence observations.

\section{ACKNOWLEDGEMENTS}

This research is supported by the Chinese PANDA International Polar Year project and the Polar Research Institute of China. The authors wish to thank all the members of the 2008/2009/2010 PRIC Dome A expeditions for their heroic efforts in reaching the site and for providing invaluable assistance to the expedition astronomers in setting up the PLATO observatory and its associated instrument suite. This research is financially supported by the US National Science Foundation and the United States Antarctic Program. The operation of PLATO at Dome A is supported by the Australian Research Council, the Australian Antarctic Division, and the University of New South Wales.

\section{REFERENCES}

[1] Moore, A., Allen, G., Aristidi, E., Ashley, M., Bedding, T., Beichman, C., Briguglio, R., Busso, M., Candidi, M., Ciardi, D., Cui, X., Cutispoto, G., Distefano, E., Espy, P., Everett, J., Feng, L., Hu, J., Jiang, Z., Kenyon, S., Kulesa, C., Lawrence, J., Le Roux, B., Leslie, T., Li, Y., Luong-Van, D., Phillips, A., Qin, W., Ragazzoni, R., Riddle, R., Sabbatini, L., Salinari, P., Saunders, W., Shang, Z., Stello, D., Storey, J., Sun, B., Suntzeff, N., Taylor, M., Tosti, G., Tothill, N., Travouillon, T., Van Belle, G., Von Braun, K., Wang, L., Yan, J., Yang, H., Yuan, X., Zhu, Z. and Zhou, X., "Gattini: a multisite campaign for the measurement of sky brightness in Antarctica", Proc. SPIE, 7012, 701226-701226-10 (2008).

[2] Yang, H., Allen, G., Ashley, M. C. B., Bonner, C. S., Bradley, S., Cui, X., Everett, J. R., Feng, L., Gong, X., Hengst, S., Hu, J., Jiang, Z., Kulesa, C. A., Lawrence, J. S., Li, Y., Luong-Van, D., McCaughrean, M. J., Moore, A. M., Pennypacker, C., Qin, W., Riddle, R., Shang, Z., Storey, J. W. V., Sun, B., Suntzeff, N., Tothill, N. F. H., Travouillon, T., Walker, C. K., Wang, L., Yan, J., Yang, J., York, D., Yuan, X., Zhang, X., Zhang, Z., Zhou, X. and Zhu, Z., "The PLATO Dome A Site-Testing Observatory: Instrumentation and First Results", Publ. Astron. Soc. Pac., 121, 174-184 (2009)

[3] Geoff Sims, Michael C. B. Ashley, Xiangqun Cui, Jon R. Everett, LongLong Feng, Xuefei Gong, Shane Hengst, Zhongwen Hu, Jon S. Lawrence, Daniel M. Luong-van, Zhaohui Shang, John W. V. Storey, Lifan Wang, Huigen Yang, Ji Yang, Xu Zhou, Zhenxi Zhu, "Optical sky brightness at Dome A, Antarctica, from the Nigel experiment", Proc. SPIE

[4] Ejiri, M., M. Okada and S. Okano, "All sky imager observation of aurora and airglow at South Pole Station, Antarctic", Journal of the United States, XXXII, 5, 202-203, 1997.

[5] Moore, Anna M.; Martin, Christopher; Maitless, Noam C.; Travouillon, Tony, "ACWI: an experiment to image the Cosmic Web from Antarctica", Proc. SPIE, 7012, pp. 70122A-70122A-11 (2008).

[6] Yuan, Xiangyan; Cui, Xiangqun; Liu, Genrong; Zhai, Fengxiang; Gong, Xuefei; Zhang, Ru; Xia, Lirong; Hu, Jingyao; Lawrence, J. S.; Yan, Jun; Storey, John W. V.; Wang, Lifan; Feng, Longlong; Ashley, Michael C. B.; Zhou, Xu; Jiang, Zhaoji; Zhu, Zhenxi, "Chinese Small Telescope ARray (CSTAR) for Antarctic Dome A", Proc. SPIE, 7012, pp. 70124G-70124G-8 (2008). 